\title{
Initial and Final Value Theorem on Fractional Hankel Transform
}

\author{
${ }^{1}$ A.S.Gudadhe, ${ }^{2}$ R.D.Taywade, ${ }^{3}$ V.N.Mahalle \\ ${ }^{I}$ Deptt.of Mathematics, Govt. Vidarbha Institute of Science \& Humanities, Amravati. \\ ${ }^{2}$ Deptt.of Mathematics, Prof. Ram Meghe Institute of Technology \& Research,Badnera, Amravati. \\ ${ }^{3}$ Deptt.of Mathematics, Bar. R.D.I.K.N.K.D. College, Badnera, Amravati.
}

Abstract: The fractional Hankel transform which is a generalization of the Hankel transform has many applications. In this paper we establish the initial and final value theorem for the generalized fractional Hankel transform.

Keywords: Fourier transform, Hankel transform, fractional Hankel transform.

\section{Introduction:}

Namias [1, 2] introduced the concept of Fourier transform of fractional order by the method of eigen values and open the path of defining the number of fractional integral transform having applications in quantum mechanics [1], Optics [3] and Signal processing [4]. Hankel transform is also generalized to its fractional version. Namias [2] himself has presented the formula for fractional Hankel transform as

Which is a generalization of Hankel transform,

$$
H_{\propto}[f(x)](y)=\frac{e^{-i(v+1)\left(\frac{\pi}{2}-\frac{\alpha}{2}\right)}}{\sin \frac{\alpha}{2}} \int_{0}^{\infty} x e^{-\frac{i}{2}\left(x^{2}+y^{2}\right) \cot \frac{\alpha}{2}} J_{v}\left(\frac{x y}{\sin \frac{\alpha}{2}}\right) f(x) d x,
$$

Kerr [5] introduced the fractional Hankel transform as,

$$
H_{\alpha}[f(x)](y)=\int_{o}^{\infty} x f(x) J_{v}(x y) d x .
$$

$$
H_{\propto}[f(x)](y)=F(y)=\int_{o}^{\infty} f(x) K_{\propto}(x, y) d x,
$$

where $K_{\alpha}(x, y)=A_{v, \alpha} e^{-\frac{i}{2}\left(x^{2}+y^{2}\right) \cot \frac{\alpha}{2}}\left(\frac{x y}{\left|\sin \frac{\alpha}{2}\right|}\right)^{\frac{1}{2}} J_{v}\left(\frac{x y}{\left|\sin \frac{\alpha}{2}\right|}\right)$

$$
=\delta(x-y) \text { for } \propto=0 \& \propto=2 \pi \text {, }
$$

and $A_{v, \alpha}=\left|\sin \frac{\alpha}{2}\right|^{-\frac{1}{2}} e^{\left(i\left(\frac{\pi}{2} \widehat{\alpha}-\frac{\alpha}{2}\right)(v+1)\right)}, \widehat{\alpha}=\operatorname{sgn} \propto, f \in L^{2}\left(R^{+}\right), \propto \in R$ and $v>-1$,

which is a generalization of the Hankel transform,

$$
H_{\propto}[f(x)](y)=\int_{o}^{\infty} \sqrt{x y} f(x) J_{v}(x y) d x .
$$

We have extended (1.1) to the Zemanian spaces and obtained inverse in [6, 7]. For the parameter $\propto=\pi$ the fractional Hankel transform reduces to the above Hankel transform. Initial and final value theorems are proved for Hankel type transformation in [8]. The paper is organized as follows. In section II, Initial value theorem and in section III final value theorem on fractional Hankel transform are given, where as section IV concludes the paper.

\section{Initial Value Theorem:}

We first prove the classical initial-value theorem.

Theorem: 2.1:

Let $|v|<\frac{1}{2}$ and a function $f(x)$ satisfies the following conditions,

i) $f(x) \rightarrow 0$ as $x \rightarrow \infty$

ii) $x^{-v-\frac{1}{2}} f(x)$ is absolutely integrable on every compact subsets $X<x<\infty$ and

iii) $\lim _{\mathrm{x} \rightarrow 0} x^{-v-\frac{1}{2}} f(x)=\rho$.

Suppose $F(y)=H^{\propto}[f(x)](y)$ is fractional Hankel transform of $f(x)$ then

$$
\lim _{\mathrm{y} \rightarrow \infty} e^{-\frac{i}{2} y^{2} \tan \frac{\alpha}{2}} y^{-v-\frac{1}{2}} F(y)=\rho B_{v, \alpha,} \text { where } B_{v, \alpha}=\frac{\sqrt{\sin \frac{\alpha}{2}}}{\left(\cos \frac{\alpha}{2}\right)^{v+1}}
$$

Proof:- Consider $\left|e^{-\frac{i}{2} y^{2} \tan \frac{\alpha}{2}} y^{-v-\frac{1}{2}} F(y)-\rho B_{v, \alpha}\right|=I$, then using equation (1.1), and the result from[9], (p. no. 30.(8.6), result (12)), we obtain, 


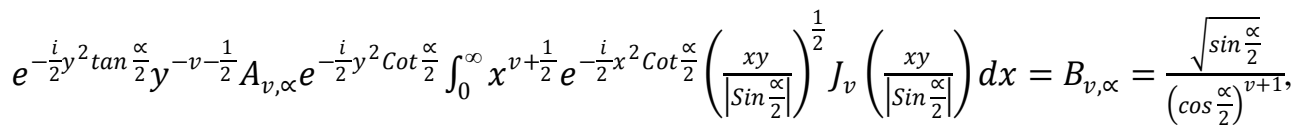

which gives,

$\mathrm{I}=\mid e^{-\frac{i}{2} y^{2} \tan \frac{\alpha}{2}} y^{-v-\frac{1}{2}} A_{v, \alpha} \int_{0}^{\infty} e^{-\frac{i}{2}\left(x^{2}+y^{2}\right) \operatorname{Cot} \frac{\alpha}{2}}\left(\frac{x y}{\operatorname{Sin} \frac{\alpha}{2}}\right)^{\frac{1}{2}} J_{v}\left(\frac{x y}{\left.\operatorname{Sin} \frac{\alpha}{2}\right)}\right) f(x) d x-$

$\rho e-i 2 y 2 \tan \alpha 2 y-v-12 \quad A v, \alpha e-i 2 \quad y 2 \operatorname{Cot} \alpha 2000 x v+12 \quad e-i 2 \quad x 2 \operatorname{Cot} \alpha 2 x y \operatorname{Sin} \alpha 212 J v(x y \operatorname{Sin} \alpha 2) d x$,

$$
\begin{aligned}
& \text { where } 0 \leq \alpha \leq \pi \text { and }-1<\operatorname{Rev}<\frac{1}{2} \\
& =\left|e^{-\frac{i}{2} y^{2} \tan \frac{\alpha}{2}} y^{-v-\frac{1}{2}} A_{v, \alpha} \int_{0}^{\infty} e^{-\frac{i}{2}\left(x^{2}+y^{2}\right) \cot \frac{\alpha}{2}}\left(\frac{x y}{\operatorname{Sin} \frac{\alpha}{2}}\right)^{\frac{1}{2}} J_{v}\left(\frac{x y}{\operatorname{Sin} \frac{\alpha}{2}}\right)\left[f(x)-\rho x^{v+\frac{1}{2}}\right] d x\right| \\
& \leq \quad\left|e^{-\frac{i}{2} y^{2} \tan \frac{\alpha}{2} y^{-v-\frac{1}{2}}} A_{v, \alpha} \int_{0}^{X} e^{-\frac{i}{2}\left(x^{2}+y^{2}\right) \operatorname{Cot} \frac{\alpha}{2}}\left(\frac{x y}{\operatorname{Sin} \frac{\alpha}{2}}\right)^{\frac{1}{2}} J_{v}\left(\frac{x y}{\operatorname{Sin} \frac{\alpha}{2}}\right)\left[f(x)-\rho x^{v+\frac{1}{2}}\right] d x\right|+
\end{aligned}
$$

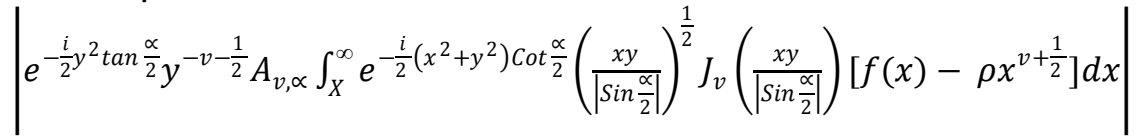

$$
\begin{aligned}
& \leq I_{1}+I_{2} \text { for some } \mathrm{X}, 0<X<\infty \text {. }
\end{aligned}
$$

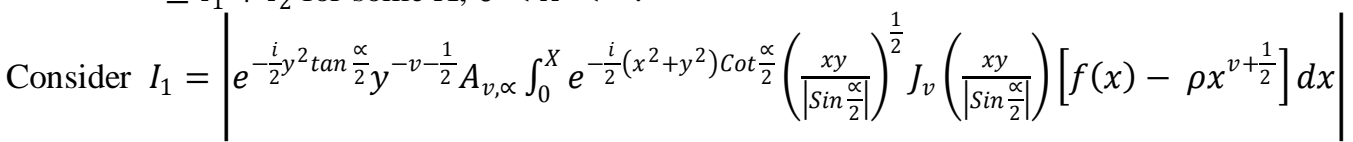$$
\leq\left|y^{-v-\frac{1}{2}} A_{v, \alpha} \int_{0}^{X}\left(\frac{x y}{\operatorname{Sin} \frac{\alpha}{2} \mid}\right)^{\frac{1}{2}} J_{v}\left(\frac{x y}{\left|\operatorname{Sin} \frac{\alpha}{2}\right|}\right) 0<x<X\left[\frac{f(x)}{x^{v+\frac{1}{2}}}-\rho\right] x^{v+\frac{1}{2}} d x\right| \text {. }
$$

By hypothesis (iii) as $\mathrm{x} \rightarrow 0,\left|\frac{f(x)}{x^{v+\frac{1}{2}}}-\rho\right|<\epsilon$.

Moreover $J_{v}(x)=O\left(x^{v}\right)$ as $x \rightarrow 0$.

Therefore above inequality becomes,

$$
\begin{gathered}
I_{1} \leq\left|y^{-v-\frac{1}{2}} A_{v, \alpha} \in \int_{0}^{X} M\left(\frac{x y}{\left|\sin \frac{\alpha}{2}\right|}\right)^{\frac{1}{2}}\left(\frac{x y}{\left|\sin \frac{\alpha}{2}\right|}\right)^{v} x^{v+\frac{1}{2}} d x\right| . \\
\leq\left|A_{v, \alpha} \epsilon \frac{M}{\left|\sin \frac{\alpha}{2}\right|^{v+\frac{1}{2}}} \int_{0}^{X} x^{2 v+1} d x\right|,
\end{gathered}
$$

integral on R.H.S. exists as $x \rightarrow 0$ if $v>-1$.

Therefore, because of R.H.S. is independent of $\mathrm{y}, I_{1}$ can be made less than $\frac{\epsilon}{2}$ if $v>-1$.

$$
\begin{aligned}
& \text { Now } I_{2}=\left|e^{-\frac{i}{2} y^{2} \tan \frac{\alpha}{2}} y^{-v-\frac{1}{2}} A_{v, \alpha} \int_{X}^{\infty} e^{-\frac{i}{2}\left(x^{2}+y^{2}\right) \operatorname{Cot} \frac{\alpha}{2}}\left(\frac{x y}{\operatorname{Sin} \frac{\alpha}{2}}\right)^{\frac{1}{2}} J_{v}\left(\frac{x y}{\operatorname{Sin} \frac{\alpha}{2}}\right)\left[f(x)-\rho x^{v+\frac{1}{2}}\right] d x\right| \\
& =\left|y^{-v-\frac{1}{2}} A_{v, \alpha} \int_{X}^{\infty}\left(\frac{x y}{\sin \frac{\alpha}{2}}\right)^{\frac{1}{2}} J_{v}\left(\frac{x y}{\operatorname{Sin} \frac{\alpha}{2}}\right)\left[f(x)-\rho x^{v+\frac{1}{2}}\right] d x\right| .
\end{aligned}
$$

Since $\sqrt{z} J_{v}(z)$ is bounded on $0<z<\infty$, moreover $\left[f(x)-\rho x^{v+\frac{1}{2}}\right]$ is absolutely integrable in $X<x<\infty$, hence $I_{2}$ can be made $<\frac{\epsilon}{2}$ as $y \rightarrow \infty$ provided $v>-\frac{1}{2}$.

Therefore $I_{2}<\frac{\epsilon}{2}$ if $v>-\frac{1}{2}$.

We get $I \leq I_{1}+I_{2}<\frac{\epsilon}{2}+\frac{\epsilon}{2}$ if $|v|<\frac{1}{2}$, from (2.1), (2.2), and (2.3).

Hence the proof. 


\section{Final Value Theorem:}

Theorem: 3.1: Let $|v|<\frac{1}{2}$ and a function $f(x)$ satisfies the following conditions,

i) $f(x) \rightarrow 0$ as $x \rightarrow \infty$

ii) $x^{v+\frac{1}{2}} f(x)$ is absolutely integrable on every compact subsets $\mathrm{X} \leq \mathrm{x}<\infty$ and

iii) $\lim _{\mathrm{x} \rightarrow \infty} x^{-v-\frac{1}{2}} f(x)=\delta$.

Suppose $F(y)=H^{\alpha}[f(x)](y)$ is fractional Hankel transform of $f(x)$ then

$$
\lim _{\mathrm{y} \rightarrow 0} e^{-\frac{i}{2} y^{2} \tan \frac{\alpha}{2}} y^{-v-\frac{1}{2}} F(y)=\delta B_{v, \alpha}
$$

Proof :- Consider

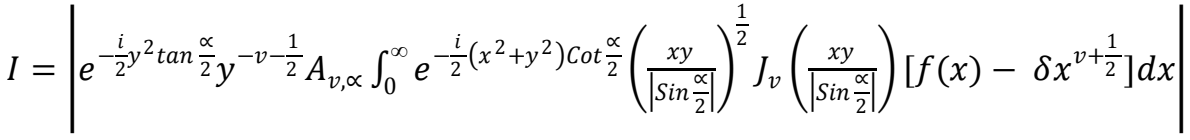

$$
\begin{aligned}
& \text { where } 0 \leq \alpha \leq \pi \text { and }-1<\operatorname{Rev}<\frac{1}{2} \\
& \leq \quad\left|e^{-\frac{i}{2} y^{2} \tan \frac{\alpha}{2}} y^{-v-\frac{1}{2}} A_{v, \alpha} \int_{0}^{X} e^{-\frac{i}{2}\left(x^{2}+y^{2}\right) \cot \frac{\alpha}{2}}\left(\frac{x y}{\sin \frac{\alpha}{2} \mid}\right)^{\frac{1}{2}} J_{v}\left(\frac{x y}{\left.\sin \frac{\alpha}{2}\right)}\right)\left[f(x)-\delta x^{v+\frac{1}{2}}\right] d x\right|+ \\
& \left|e^{-\frac{i}{2} y^{2} \tan \frac{\alpha}{2}} y^{-v-\frac{1}{2}} A_{v, \alpha} \int_{X}^{\infty} e^{-\frac{i}{2}\left(x^{2}+y^{2}\right) \operatorname{Cot} \frac{\alpha}{2}}\left(\frac{x y}{\left|\operatorname{Sin} \frac{\alpha}{2}\right|}\right)^{\frac{1}{2}} J_{v}\left(\frac{x y}{\left|\operatorname{Sin} \frac{\alpha}{2}\right|}\right)\left[f(x)-\delta x^{v+\frac{1}{2}}\right] d x\right|, \\
& \leq I_{1}+I_{2} \text {. }
\end{aligned}
$$

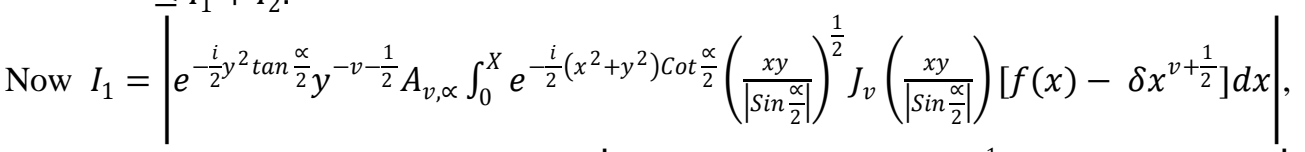
moreover $J_{v}(z)=O\left(z^{v}\right)$ as $z \rightarrow 0, I_{1}=\left|y^{-v-\frac{1}{2}} A_{v, \alpha} \int_{0}^{X} M\left(\frac{x y}{\left|\sin \frac{\alpha}{2}\right|}\right)^{v+\frac{1}{2}}\left[f(x)-\delta x^{v+\frac{1}{2}}\right] d x\right|$

$$
=\left|\frac{A_{v, \alpha}}{\left|\sin \frac{\alpha}{2}\right|^{v+\frac{1}{2}}} \int_{0}^{X} x^{v+\frac{1}{2}}\left[f(x)-\delta x^{v+\frac{1}{2}}\right] d x\right|
$$

The term is independent of $\mathrm{y}$ and $x^{v+\frac{1}{2}} f(x)$ is integrable according to condition (ii),

therefore integral converges if $x^{2 v+2}$ converges as if $v>-1$, hence $I_{1}$ can be made less than $\frac{\epsilon}{2}$.

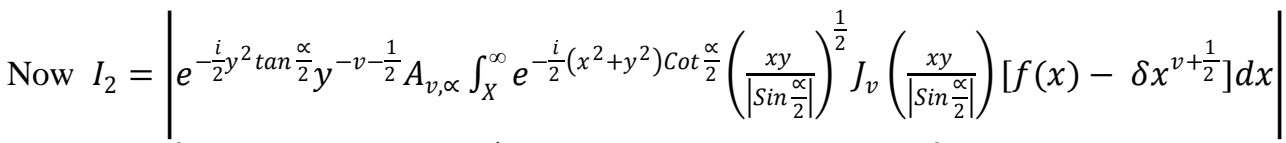

$$
=\left|y^{-v-\frac{1}{2}} A_{v, \propto} \int_{X}^{\infty}\left(\frac{x y}{\left|\sin \frac{\alpha}{2}\right|}\right)^{\frac{1}{2}} J_{v}\left(\frac{x y}{\left|\sin \frac{\alpha}{2}\right|}\right)\left[f(x)-\delta x^{v+\frac{1}{2}}\right] d x\right|,
$$

but $\sqrt{z} J_{v}(z)=0(1)$ as $z \rightarrow \infty$

$$
I_{2} \leq\left|y^{-v-\frac{1}{2}} A_{v, \alpha} M \int_{X}^{\infty} x^{v+\frac{1}{2}}\left[\frac{f(x)}{x^{v+\frac{1}{2}}}-\delta\right] d x\right|,
$$

by condition (iii) in hypothesis, R.H.S. converges in $X \leq x<\infty$. Also as $y \rightarrow 0$, R.H.S. converges if $v<-\frac{1}{2}$, therefore $I \leq I_{1}+I_{2}<\frac{\epsilon}{2}+\frac{\epsilon}{2}$ provided $-1<v<-\frac{1}{2}$, using (3.1), (3.2) and (3.3),

Hence the proof.

\section{Conclusion:}

We have proved the Initial and Final value theorems for fractional Hankel transform. Further we plan to apply these theorems for solving some definite integrals. 


\section{References:}

[1] Namias V., The fractional order Fourier transform and its application to quantum mechanics, J. Inst. Math. Appl., Vol. 25, 241-265, (1980).

[2] Namias V., Fractionalization of Hankel transform, J. Inst. Math. Appl., Vol. 26, 187-197, (1980).

[3] Fange,daomu Zhao and Shaomi Wang, Fractional Hankel transform and the diffraction of misaligned optical systems, J. of Modern optics, Vol. 52, No.1, 61-71,(2005).

[4] Ozaktas H. M., Zalvesky Z., Kuntay M. Alper, The fractional Fourier transform with applications in optics and signal processing (Pub. John Wiley and Sons. Ltd, 2001).

[5] Kerr F. H., A fractional power of Hankel transform, Int. J. Math. Ana. and app., Vol. 158, 114-123, (1991).

[6] Taywade R. D., Gudadhe A. S., Mahalle V. N., On generalzed fractional Hankel transform, Int. J. of Math. Ana., Vol. 6, no. 17-20, (2012).

[7] Taywade R. D., Gudadhe A. S., Mahalle V. N., Inversion of fractional Hankel transform in the Zemanian space, Proc. ICBEST, Int. J. Comp. App., 31-34,(2012).

[8] Chaudhary M. S. and Thorat S.P., Abelian theorem related to the Hankel type transformation, J. Indian Acad. Math. Vol. 21 , no. 1, 21 29, (1999).

[9] Bateman Harry, Tables of integral transforms, Vol.II (MacGraw-Hill book company Inc., New York. 1954). 